\title{
Percutaneous endoscopic gastrostomy for patients requiring for long-term enteral nutrition: A retrospective study of 84 patients.
}

\author{
Chunli Cao ${ }^{1 \#}$, Chunli Cong ${ }^{1 \#}$, Yue Li $^{2}$, Yan Meng' ${ }^{2}$, Shirong Kang ${ }^{\text {* }}$ \\ ${ }^{1}$ Department of Gastroenterology, First Affiliated Hospital of Inner Mongolia Medical University, Huhhot, PR China \\ ${ }^{2}$ Department of Gastroenterology, Guangdong Provincial Key Laboratory of Gastroenterology, Nanfang Hospital, \\ Southern Medical University, Guangzhou, PR China \\ ${ }^{3}$ Department of Thoracic Surgery, First Affiliated Hospital of Inner Mongolia Medical University, Huhhot, PR China

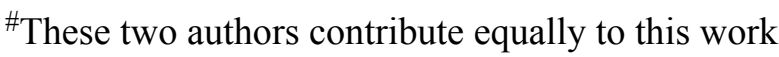

\begin{abstract}
Background and aims: Existed studies about Percutaneous Endoscopic Gastrostomy (PEG) were mainly aimed at certain patient populations and data came from China are quite limited. Our aim was to investigate whether PEG in South China is effective and to compare the indications and complications of PEG with those reported in the literature.

Materials and methods: Patients underwent PEG procedure during the period from January 2000 to October 2014 in Nanfang Hospital were retrospectively collected and analysed. The patients' lymphocyte counts, leukocyte counts, serum albumin, hemoglobin, serum total protein, weight and BMI before and after PEG operation were reviewed as the main outcome measurement. Indications, complications and success rates of PEG procedure were also evaluated.

Results: A total of 84 patients (61 males, 23 females) were enrolled, with an average age of $55.01 \pm 15.16$ $y$ (range 3.0-92 y). The overall success rate is about 98.8\%. The most common indication for PEG in South China is head and neck cancer $(27 / 84,32.1 \%)$. Significant differences were observed in parameters such as lymphocyte counts, serum albumin, serum total protein, body weight, BMI before and after PEG operation.(1.29 vs. 1.76, $p=0.005 ; 33.58$ vs. 33.66, $p=0.012 ; 62.82$ vs. 67.29, $p=0.018 ; 43.62$ vs. 48.29, $\mathrm{p}=0.044 ; 16.24$ vs. $18, \mathrm{p}=0.030$; respectively). The most common procedure-related complication was PEG tube removal $(6 / 84,7.14 \%)$, which were all well managed.

Conclusions: This is the first retrospective case series evaluating PEG procedure in South China. Our results indicate that PEG is an effective and safe method for long-term enteral nutrition.
\end{abstract}

Keywords: PEG, Enteral nutrition, Indication, Complication.

Accepted on August 17, 2017

\section{Introduction}

Percutaneous Endoscopic Gastrostomy (PEG) is an endoscopic medical procedure in which a tube (PEG tube) is passed into a patient's stomach through the abdominal wall, most commonly to provide a means of feeding when oral intake is no longer possible or adequate [1]. Since the first report of PEG in 1980 by Gauderer [2], the procedure has rapidly become widely used routine practice. Even though PEG is one of the most common endoscopic procedures performed worldwide, PEG tubes are sometimes placed inappropriately in patients unable to tolerate adequate oral intake because of incorrect and unrealistic understanding of their indications, contraindications and complications. In addition, although generally considered to be a safe procedure, there are still many reported minor and major complications for PEG [3-6]. Awareness of these potential complications can improve the quality of management for patients with need of PEG tube insertion. Large numbers of studies have evaluated PEG procedure in certain patient population, such as children [7], trauma patients [8], ICU patients [9], head and neck cancer patients [10], which all proved that PEG a safe and effective technique for those kinds of patients. However, existed studies are mainly aimed at various patient populations and data about PEG came from the district of South China are quite limited. Therefore, we performed this current study in order to investigate whether PEG procedure in South China is effective and to compare the indications, complications of PEG with those reported in the other literature.

\section{Materials and Methods}

We retrospectively reviewed the medical records of 115 patients underwent PEG procedure during the period from 
January 2000 to October 2014 in Nanfang Hospital. A total of 84 subjects fulfilled our criteria and were enrolled in this current study. The inclusion criteria were a history of PEG operation and a follow-up of at least 1 month after the procedure. The collected parameters included patients' age, sex, lymphocyte counts, leukocyte counts, serum albumin, hemoglobin, serum total protein, weight, Body Mass Index (BMI) before and after PEG operation. The indication for PEG placement, the success or failure of PEG insertion, the number and type of complications and their management were also recorded. All procedures were performed by experienced endoscopists using the standard pull method, and under general anesthesia as described by Gauderer [2]. This study was approved by Nanfang Hospital Institutional Review Board. All patients were informed of the benefits and risks of the procedure and signed the written informed consent document prior to the procedure.

\section{Statistical analysis}

Data were expressed as the mean \pm SD for normally distributed results. Independent sample t-test was used to analysis measurement data before and after PEG operation. All analyses were performed using SPSS 13.0 (IBM, Armonk, NY, USA). Values with $\mathrm{P}<0.05$ were considered statistically significant in all cases.

\section{Results}

Our study included 84 patients (61 males, 23 females), with an average age of $55.01 \pm 15.16$ years (range 3.0-92 y). Forty-six patients $(54.8 \%)$ had low albumin level and 64 patients $(72.6 \%)$ had low BMI, indicating that most patients enrolled were accompanied by a poor nutritional status before PEG procedure (Table 1). None of these patients had preoperative contraindication to PEG placement such as coagulopathy, previous gastrectomy, ascites or morbid obesity. The patients suffered from a variety of diseases which generally can be classified into four major categories including neurological diseases and psychomotor retardation, reduced level of consciousness, cancer and miscellaneous. Among these indications, caner especially head and neck cancer was the most common (Table 2). PEG placement was possible and successful in 83 patients $(98.8 \%)$ except one for the patient's anterior wall of insufflated stomach could not come in contact with the abdominal wall, leading to the failure of the intervention. During the period of follow-up after the PEG operation, we observed great improvement on the related observational index concerning about patient's nutrition. There were significant differences in parameters such as lymphocyte counts, serum albumin, serum total protein, weight and BMI before and after PEG operation.(1.29 vs. $1.76, \mathrm{p}=0.005 ; 33.58$ vs. $33.66, \mathrm{p}=0.012 ; 62.82$ vs. $67.29, \mathrm{p}=0.018 ; 43.62$ vs. 48.29 , $\mathrm{p}=0.044$; 16.24 vs. 18, $\mathrm{p}=0.030$; respectively) (Figure 1). Additionally, the most common procedure-related complication in this present study was PEG tube removal $(6 / 83,7.2 \%)$ and other complications include wound infection $(3 / 83,3.6 \%)$, hemorrhage $(2 / 83,2.4 \%)$ and tube blockage $(1 / 83,1.2 \%)$, which were all well managed (Table 3 ).

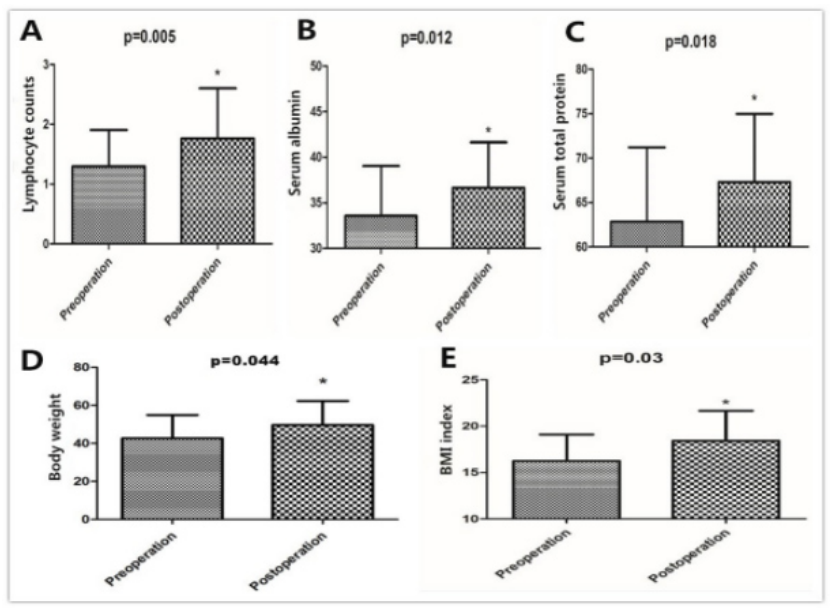

Figure 1. Changes of laboratory characteristics before and after PEG operation. Significant differences were observed in parameters such as the patients' lymphocyte counts (A), serum albumin (B), serum total protein (C), body weight (D), BMI (E) before and after PEG operation. $P$ value of less than 0.05 was considered statistically significant. Results are expressed as median \pm standard deviation. All these statistical analyses were performed by using the SPSS 13.0 statistical package.

Table 1. Demographic and laboratory characteristics of the study subjects $(n(\%))$.

\begin{tabular}{ll}
\hline Age $(\mathrm{y})$ & $55.0 \pm 15.2$ \\
\hline Gender, M:F & $61: 23(72.6 \%: 28.4 \%)$ \\
\hline Lymphopenia $\left(<1.1 \times 10^{9}\right)$ & $32(38.1 \%)$ \\
\hline Low hemoglobin $(\mathrm{M}<120 \mathrm{~g} / \mathrm{L}, \mathrm{F}<110 \mathrm{~g} / \mathrm{L})$ & $50(59.5 \%)$ \\
\hline Low albumin $(<3.5 \mathrm{~g} / \mathrm{dL})$ & $46(54.8 \%)$ \\
\hline Low BMI $(<18.5)$ & $64(76.2 \%)$
\end{tabular}

M: Male; F: Female; BMI: Body Mass Index.

Table 2. Indications for patients referred for insertion of a PEG tube $(n(\%))$.

\begin{tabular}{ll}
\hline Neurological disease and psychomotor retardation & $32(38.0 \%)$ \\
\hline Stroke/cerebrovascular disease & $24(28.4 \%)$ \\
\hline Motor neuron diseases (amyotrophic lateral sclerosis) & $4(4.8 \%)$ \\
\hline Parkinson's disease & $2(2.4 \%)$ \\
\hline Status epilepticus & $1(1.2 \%)$ \\
\hline Dementia & $1(1.2 \%)$ \\
\hline Cancer & $34(40.5 \%)$ \\
\hline Head and neck cancer & $27(32.1 \%)$ \\
\hline Esophageal cancer & $5(6.0 \%)$ \\
\hline Other cancer & $2(2.4 \%)$ \\
\hline Reduced level of consciousness & $14(16.7 \%)$ \\
\hline
\end{tabular}


84 patients

\begin{tabular}{ll}
\hline Head injury & $12(14.3 \%)$ \\
\hline Prolonged coma & $2(2.4 \%)$ \\
\hline Miscellaneous & $4(4.8 \%)$ \\
\hline Crohn's disease & $2(2.4 \%)$ \\
\hline Burns & $1(1.2 \%)$ \\
\hline Gastric decompression & $1(1.2 \%)$ \\
\hline
\end{tabular}

Table 3. Major complications related to PEG placement ( $n(\%))$.

\begin{tabular}{ll}
\hline PEG tube removal & $6(7.2 \%)$ \\
\hline Wound infection & $3(3.6 \%)$ \\
\hline Hemorrhage & $2(2.4 \%)$ \\
\hline Tube blockage & $1(1.2 \%)$ \\
\hline Total & $12(14.4 \%)$ \\
\hline
\end{tabular}

\section{Discussion}

Percutaneous Endoscopic Gastrostomy (PEG) administration of enteral feeding is one the most commonly used method of nutritional support when oral nutrition is no longer possible or adequate. The superiority of percutaneously placed gastrostomies compared to former surgical gastrostomy procedures (i.e. Witzel, Stamm, Janeway technique) has been shown clearly in many clinical studies [11,12]. Existed evidence have also proved that PEG was associated with a lower probability of intervention failure, suggesting the endoscopic procedure is more effective and safe as compared to nasogastric tube [13]. When patients need for short-term enteral feeding, placing a nasogastric tube may be enough. However, for those patients requiring for long-term enteral nutrition, PEG should be considered. Large numbers of studies have been carried out to evaluate the effectiveness and safety of PEG in various kinds of patients. However, data about PEG came from China are quite scarce. In our study, we investigated and evaluated PEG procedure performed in South China for the first time. PEG procedure may be indicated in a wide range of diseases: neurological diseases, reduced level of consciousness, cancer and miscellaneous. In this present study, cancer $(40.5 \%)$ especially head and neck cancer $(32.1 \%)$ was the most common indication for PEG procedure, which is different from the previous literature that the main therapeutic indications are benign neurological disorders. Gardine et al. [14] found that advanced cancer stage (mainly stage IV), primary pharyngeal tumors, combined treatment of surgery and radiotherapy and preoperative weight loss of more than 10 pounds were associated with the need for PEG insertion for head and neck cancer. Among the 27 patients indicated by head and neck cancer in our study, most patients $(23 / 27,85.2 \%)$ are nasopharyngeal tumors combined with treatment of radiotherapy. The reason for this phenomenon may be that head and neck cancer especially nasopharyngeal carcinoma was much more prevalent in region of Guangdong Province in South China [15].
Significant differences were observed in parameters such as the patients' lymphocyte counts, serum albumin, serum total protein, body weight, BMI before and after PEG operation. This result indicated that PEG can prevent the further weight loss and improve the patients' nutritional status which is consistent with findings reported by previous studies. The most common procedure-related complication in this present study was inadvertent PEG tube removal (7.2\%) and the other complications included wound infection, hemorrhage and tube blockage, which were all managed by endoscopic adjustment or reinstallation or antibiotic treatment. To our knowledge, this is the largest case series evaluated and compared PEG procedure performed in South China with other literature. In conclusion, there is low probability of intervention failure for PEG procedure $(83 / 84,98.81 \%)$ and high subjective comfort for patient. In addition, the feeding efficacy of PEG is satisfactory and there is low procedure-related complication. From the results above mentioned, PEG is an effective and safe method for long-term enteral nutrition However, further studies are needed to evaluate PEG feeding in certain patient populations, such as those with diabetes or advanced dementia and in old patients aged more than 80 years for there are still uncertain benefits for those kinds of patients.

\section{Ethics Committee Approval}

Ethics committee approval was received for this study from the ethics committee of First Affiliated Hospital of Inner Mongolia Medical University.

\section{Informed Consent}

N/A

\section{Peer-Review}

Externally peer-reviewed

\section{Author Contributions}

Chunli Cao and Chunli Cong designed the study. Yue Lian and Yan Meng recorded and analysed the data. Shirong Kang drafted the manuscript. All authors have read and approved the submitted version of the paper.

\section{Conflict of Interest}

The authors declare no conflict of interest.

\section{Financial Disclosure}

The authors declared that this project was supported by Guangdong Province Universities and Colleges Pearl River Scholar Funded Scheme support.

\section{References}

1. Loser C, Aschl G, Hebuterne X. ESPEN guidelines on artificial enteral nutrition-percutaneous endoscopic 
gastrostomy (PEG). Clin Nutr (Edinburgh, Scotland) 2005; 24: 848-861.

2. Gauderer MW, Ponsky JL, Izant RJ. Gastrostomy without laparotomy: a percutaneous endoscopic technique. J Pediatr Surg 1980; 15: 872-875.

3. Santos-Antunes J, Nunes AC, Cardoso H. Gastric outlet obstruction following percutaneous endoscopic gastrostomy. Endoscopy 2014; 46: 445-446.

4. Than MM, Witherspoon J, Tudor G. Gastric outlet obstruction secondary to percutaneous endoscopic gastrostomy tube placement. Endoscopy 2012; 44: 269-270.

5. Fischer LS, Bonello JC, Greenberg E. Gastrostomy tube migration and gastric outlet obstruction following percutaneous endoscopic gastrostomy. Gastrointest Endosc 1987; 33: 381-382.

6. Schmidt C, Gerdes H, Hawkins W. A prospective observational study examining quality of life in patients with malignant gastric outlet obstruction. Am J Surg 2009; 198: 92-99.

7. Avitsland TL, Kristensen C, Emblem R. Percutaneous endoscopic gastrostomy in children: a safe technique with major symptom relief and high parental satisfaction. J Pediatr Gastroenterol Nutr 2006; 43: 624-628.

8. Dwyer KM, Watts DD, Thurber JS. Percutaneous endoscopic gastrostomy: the preferred method of elective feeding tube placement in trauma patients. J Trauma 2002; 52: $26-32$

9. Zippi M, Fiorani S, De Felici I. Percutaneous endoscopic gastrostomy (PEG) in critically ill patients performed at bed in Intensive Care Unit: report of our experience. La Clinica Terapeutica 2009; 160: 359-362.

10.Zuercher BF, Grosjean P, Monnier P. Percutaneous endoscopic gastrostomy in head and neck cancer patients: indications, techniques, complications and results. Eur Feder Oto Rhino Laryngol Soc German Soc Otorhinolaryngol Head Neck Surg 2011; 268: 623-629.

11. Grant JP. Comparison of percutaneous endoscopic gastrostomy with Stamm gastrostomy. Ann Surg 1988; 207: 598-603.

12. Ho CS, Yee AC, McPherson R. Complications of surgical and percutaneous nonendoscopic gastrostomy: review of 233 patients. Gastroenterology 1988; 95: 1206-1210.

13. Gomes CA, Lustosa SA, Matos D. Percutaneous endoscopic gastrostomy versus nasogastric tube feeding for adults with swallowing disturbances. Cochrane Datab Sys Rev 2012; 3: 8096

14. Gardine RL, Kokal WA, Beatty JD. Predicting the need for prolonged enteral supplementation in the patient with head and neck cancer. Am J Surg 1988; 156: 63-65.

15. Yu MC, Yuan JM. Epidemiology of nasopharyngeal carcinoma. Semin Cancer Biol 2002; 12: 421-429.

\section{*Correspondence to}

Shirong Kang

Department of Thoracic Surgery

First Affiliated Hospital of Inner Mongolia Medical University

PR China 